\title{
Translocation of double-strand DNA through a silicon oxide nanopore
}

\author{
A. J. Storm, ${ }^{1}$ J. H. Chen, ${ }^{1,2}$ H. W. Zandbergen, ${ }^{1}$ and C. Dekker ${ }^{1}$ \\ ${ }^{1}$ Kavli Institute of Nanoscience, Delft University of Technology, Lorentzweg 1, 2628 CJ Delft, The Netherlands \\ ${ }^{2}$ Netherlands Institute for Metals Research, 2628 AL Delft, The Netherlands
}

(Received 16 August 2004; published 6 May 2005)

\begin{abstract}
We report double-strand DNA translocation experiments using silicon oxide nanopores with a diameter of about $10 \mathrm{~nm}$. By monitoring the conductance of a voltage-biased pore, we detect molecules with a length ranging from 6557 to 48500 base pairs. We find that the molecules can pass the pore both in a straight linear fashion and in a folded state. Experiments on circular DNA further support this picture. We sort the molecular events according to their folding state and estimate the folding position. As a proof-of-principle experiment, we show that a nanopore can be used to distinguish the lengths of DNA fragments present in a mixture. These experiments pave the way for quantitative analytical techniques with solid-state nanopores.
\end{abstract}

DOI: 10.1103/PhysRevE.71.051903

PACS number(s): 87.14.Gg, 87.15.Tt

\section{INTRODUCTION}

Translocation of biopolymers such as polypeptides, DNA, and RNA is an important process in biology. Transcribed RNA molecules, for example, are transported out of the nucleus through a nuclear pore complex. Viral injection of DNA into a host cell is another example. Experimental and theoretical studies of this process have attracted considerable attention. Kasianowicz et al. [1] demonstrated that an $\alpha$-hemolysin pore in a lipid membrane can be used to study the translocation process in vitro. By measuring the ionic current through a voltage-biased nanopore, they detect individual molecules that are pulled through the pore by the electric field. More recently, Li et al. [2,3] showed that solidstate nanopores can also be used for similar experiments. Here we describe a set of translocation experiments with silicon oxide nanopores that were fabricated with a technique described earlier [4]. We present a detailed study of the translocation dynamics of double-strand DNA molecules with lengths ranging from 6557 to 48500 base pairs.

The dynamics of DNA translocation through nanopores is particularly relevant for potential analytical techniques based on nanopores. Rapid oligonucleotide discrimination on the single-molecule level has been demonstrated with $\alpha$-hemolysin [5] and more recently solid-state nanopores were used for a first study of folding effects in double-strand DNA molecules [3]. Future applications of this technique may include DNA size determination, haplotyping, and sequencing.

The use of nanopores for the detection and analysis of single molecules is inspired by the working principle of a Coulter counter [6]. Particles suspended in an electrolyte solution are drawn through a small channel, separating two reservoirs. When a particle enters the channel, it displaces its own volume of solution, thereby increasing the electrical impedance of the channel. By applying a voltage over the channel, the passing particles are detected as current drops, and the magnitude of the current dip scales with the volume of the particle. This analytical technique has proven very effective in determining the concentration and size distribution of particles, down to the submicrometer size. The availability of nanometer-sized apertures has extended this technique to the single-molecule level. Such nanopores have been shown to detect polynucleotides, using pores with a diameter slightly larger than the cross section of the molecule. One of the main challenges is to obtain pores with a diameter comparable to the diameter of the polymer ( $2 \mathrm{~nm}$ for double-strand DNA). Figure 1 shows a typical experimental layout for such experiments. During translocation, only a small fraction of the polymer is inside the sensing volume of the pore. Consequently, the duration and not the depth is a measure for the length of the polymer. It is interesting to note that apart from volumetric effects, other effects could change the conductance of the nanopore during DNA translocation. Chang et al. [7] claim that DNA translocation at low salt concentration causes an increase in conductance.

Until now, most experiments have been carried out using the $\alpha$-hemolysin pore complex. It self-assembles, and thus all pores are identical down to the atomic level. Such pores have been used most extensively, but there is also a large interest in synthetic pores. The fabrication of such pores requires an accuracy on the single-nanometer level, which is far from straightforward with existing techniques. Such an effort is justified, however, because of a number of potential advantages of solid-state pores over protein pores. First, the dimensions of the pore can be varied to ensure optimal sen-

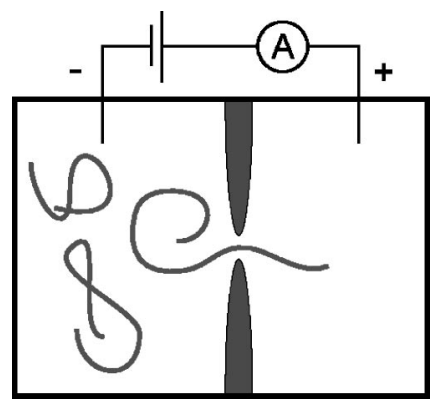

FIG. 1. Schematic layout of the experiment: A charged polymer is electrophoretically driven through a nanometer-sized aperture, located between two reservoirs kept at a potential difference. The presence of a molecule inside the pore lowers the amount of conducting solution inside the pore. Passing molecules can thus be detected as short dips in the ionic current through the pore. 
sitivity. Second, solid-state nanopores are much more robust: They are expected to have a much longer lifetime, and experiments can be performed at high temperatures and at extreme $p \mathrm{H}$ values. Finally, solid-state pores allow additional device complexity and incorporation of additional tools. For example, Li et al. [3] speculated that electrodes integrated within the nanopore could be used to detect tunneling currents through molecules as they pass the nanopore, analogous to the working principle of a scanning tunneling microscope. They aim to read off the base sequence of a single DNA molecule as it passes the detector inside the nanopore.

In this study, we report single-molecule detection of double-strand DNA using silicon oxide nanopores that were fabricated using a technique recently developed in Delft [4]. Linear double-strand DNA fragments of 11.5 kilobase pairs (kbp) and $48.5 \mathrm{kbp}$ and a circular DNA vector of $11.5 \mathrm{kbp}$ were studied in detail with our nanopore setup. We find strong evidence for translocation of folded DNA, where passage starts not at one of the ends of the molecule but somewhere in between [3]. A part of the molecule folds back onto itself, resulting in current blockades that are larger in amplitude and shorter in time than simple linear translocation events. We present additional evidence for this picture with the detection of translocation of circular DNA molecules. These circular molecules can only pass the pore in a folded state. Indeed we observe qualitatively different statistics of the translocation events compared to linear molecules that are in good agreement with the model. The experiments demonstrate that double-strand DNA with a persistence length of about $50 \mathrm{~nm}$ can indeed pass a $8 \mathrm{~nm}$ pore in a folded fashion. We chose to work with relatively large pores between 8 and $10 \mathrm{~nm}$. This was motivated by observations of Li et al. [3] that a 3-nm pore shows significantly more scatter in translocation times than a $10-\mathrm{nm}$ pore.

Finally, we present an experiment that demonstrates the remarkable sensitivity of a nanopore detector. We have used a nanopore to analyze the size distribution of a standard mixture of double-strand DNA fragments, ranging in length between $6.5 \mathrm{kbp}$ and $24 \mathrm{kbp}$. In this proof-of-principle experiment, we analyzed translocation events of about 2500 individual molecules (about $30 \mathrm{fg}=3 \times 10^{-14} \mathrm{~g}$ or 4 zeptomol $=4 \times 10^{-21} \mathrm{~mol}$ ), and demonstrate clear separation in length.

\section{MATERIALS AND METHODS}

\section{A. Fabrication of solid-state nanopores}

We use solid-state nanopores fabricated using a process reported earlier [4]. Starting from silicon-on-insulator (SOI) wafers (obtained from SOITEC, France), we first fabricate a free-standing silicon membrane using electron beam lithography, reactive-ion etching, and wet chemical $\mathrm{KOH}$ etching. Subsequently, we fabricate pores of about $50 \mathrm{~nm}$ in this 340-nm-thick silicon membrane by a second step of electronbeam lithography and wet chemical $\mathrm{KOH}$ etching. This membrane is thermally oxidized to form a 40-nm-thick, silicon dioxide layer on the surface of the membrane and pore. Surprisingly, we found that we can shrink the pore by exposure to a high dose of electrons, supplied by the imaging

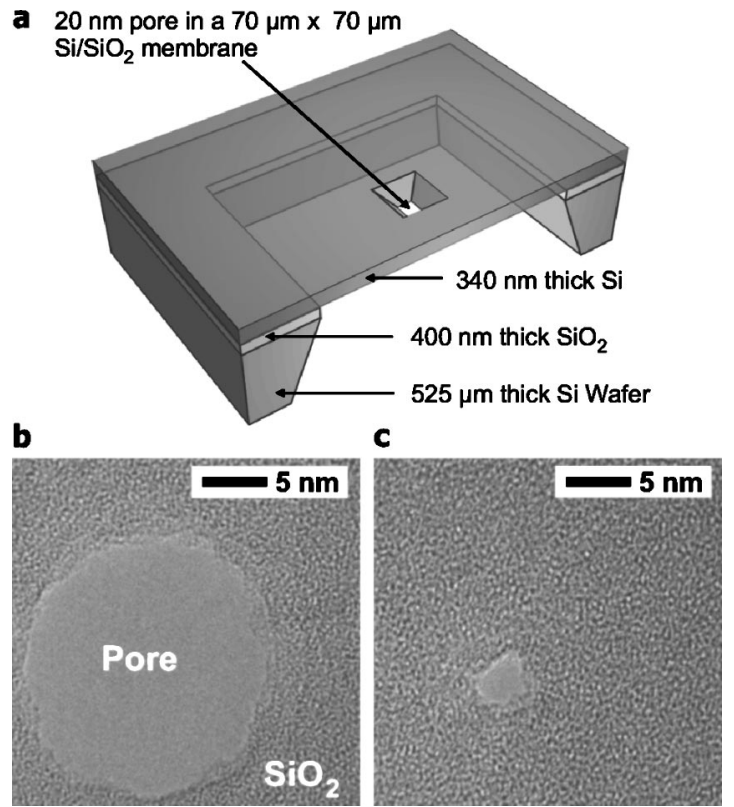

FIG. 2. (a) Cross-sectional view of our sample layout. From silicon-on-insulator (SOI) wafers we fabricate free-standing, silicon membranes with a thickness of $340 \mathrm{~nm}$. A pore is etched in this membrane by wet $\mathrm{KOH}$ etching. Thermal oxidation of all silicon surfaces yields a pore with a diameter of about $20 \mathrm{~nm}$, surrounded by $40 \mathrm{~nm}$ of silicon oxide. (b) Using the electron beam of a TEM microscope, we can effectively melt the silicon oxide causing the pore to shrink. Here we show an electron micrograph of a pore before the shrinking process. (c) Final image of a pore just before we blank the electron beam. The pore shown here has a diameter of about $3 \mathrm{~nm}$.

beam of a transmission electron microscope (TEM) equipped with a field emission gun. The process can be observed in real time on the fluorescent screen of the microscope. This allows us to stop when the desired dimensions of the pore have been obtained, by blanking the electron beam (see Fig. 2 for a schematic device layout and two electron micrographs obtained during the shrinking process). This technique allows us to very reliably fabricate solid-state nanopores with any desired diameter, down to at least $2 \mathrm{~nm}$. We used pores with a diameter between 8 and $10 \mathrm{~nm}$ for the experiments reported in this work. We estimate that the length of our nanopores is on the order of $20 \mathrm{~nm}$, based on TEM tilting experiments. The length is thus much shorter than all the DNA fragments that were studied.

\section{B. DNA material}

A 11.5-kbp "charomid" vector was cloned into ecoli dh5- $\alpha$ bacteria, cultured, and purified using a commercial Mini-prep kit (Qiagen). It was subsequently restriction digested using EcoRI and purified using phenol-chloroform extraction. The length of the digested plasmid was verified using gel electrophoresis. To obtain circular DNA of $11.5 \mathrm{kbp}$ with a single nick, the nicking enzyme N.BbvCIA was used in stead of the EcoRI. The same protocol was used to purify this fragment. Linear $\lambda$-DNA with a length of $48.5 \mathrm{kbp}$ was obtained commercially (Promega, Benelux). The standard 


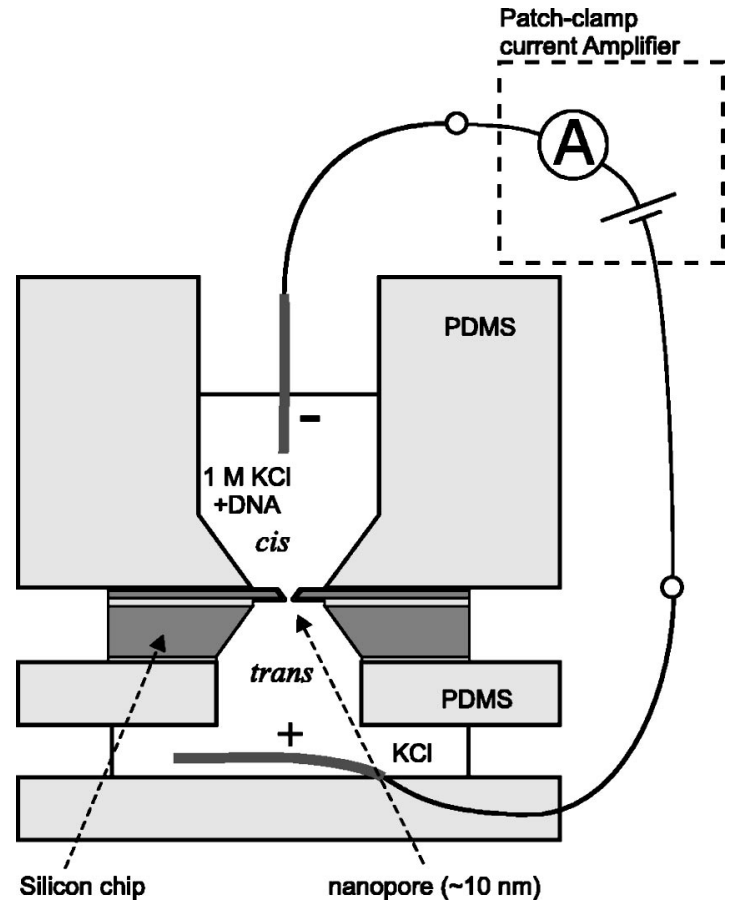

FIG. 3. Cross-sectional view of the fluidic setup (not to scale). Shown in light grey are two PDMS parts that contain the reservoirs. A silicon chip containing the nanopore is clamped horizontally in between. Both reservoirs contain a $\mathrm{Ag} / \mathrm{AgCl}$ electrode for electrical contact. A patch-clamp current amplifier is used for the ioniccurrent measurements at high bandwidth.

mixture of DNA fragments produced by incubating $\lambda$-DNA with the HindIII restriction enzyme was also purchased from the same source. It contained fragments with a length of 23130 bp, 9416 bp, 6557 bp, 4361 bp, 2322 bp, 2027 bp, $564 \mathrm{bp}$, and $125 \mathrm{bp}$.

\section{Experimental setup}

TEM-fabricated nanopores were mounted in a fluidic setup. The nanopore device is clamped horizontally in between two parts of a Poly(dimethylsiloxane) (PDMS) cell; see Fig. 3. Each of these parts contains a fluidic reservoir of about $50 \mu \mathrm{l}$, and the nanopore is the only connection between the two reservoirs. Both reservoirs contain a $\mathrm{Ag} / \mathrm{AgCl}$ electrode for electrical contact to the liquid. A patch-clamp amplifier (Axopatch 200B amplifier from Axon Instruments) was used to apply a voltage over the two electrodes and to detect and amplify the resulting ionic current. The signal is low-pass filtered using a four pole Bessel filter with a cutoff frequency of $10 \mathrm{kHz}$. The signal was then digitized (with an Axon 1322A digitizer) at $200 \mathrm{kHz}$ and recorded to the hard disk of the computer. All experiments were performed at room temperature. We also recorded the current signal continuously on a Digital Audio Tape (DAT) with a Dagan DAS-75 recorder.

A well-known difficulty of small fluidic systems is priming it with the electrolyte solution. Incomplete priming can result in trapped air bubbles inside the nanopore, blocking the ionic current partially or even completely. We found that

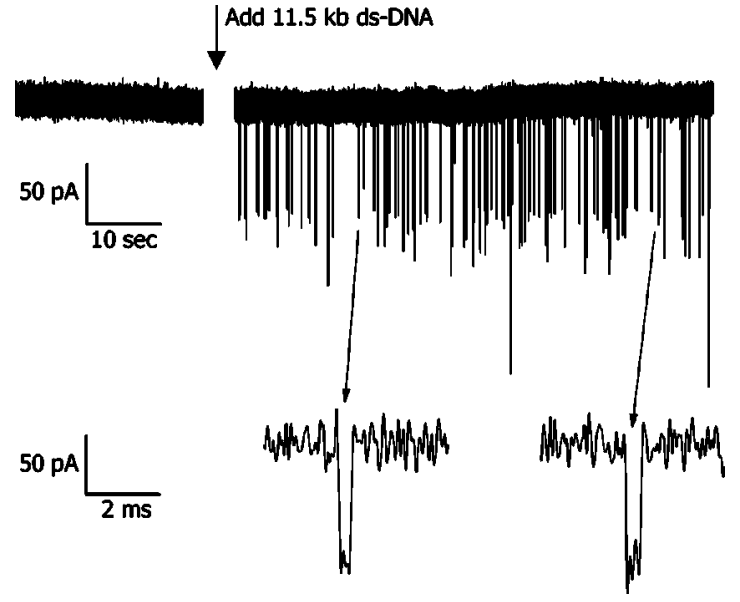

FIG. 4. Measured ionic current versus time. After addition of DNA to the cis side of the pore we clearly observe downward dips in the current. In the bottom panel, two individual events are shown at an increased time resolution.

cleaning our samples for about $30 \mathrm{~s}$ in an oxygen plasma aids in wetting of our devices. This procedure cleans the sample from organic contamination and results in a hydrophilic $\mathrm{SiO}_{2}$ surface. Immediately after the plasma treatment we mount our device in the setup and fill it with a degassed and filtered buffer solution $(1.0 M \mathrm{KCl}, 10 \mathrm{mM}$ Tris- $\mathrm{HCl}$, $p \mathrm{H}=8.0,1 \mathrm{~m} M$ EDTA). Usually this procedure leads to a stable current signal, but occasionally additional oxygenplasma cleaning was necessary before a stable, low noise signal was obtained.

\section{RESULTS}

\section{A. Current blockades}

First, we discuss the experiments performed on linear double-strand DNA molecules of $11.5 \mathrm{kbp}$ and $48.5 \mathrm{kbp}$. A 10-nm nanopore was mounted in the experimental setup and filled with the $1 M \mathrm{KCl}$ buffer solution, as described in the pervious section. After establishing a stable ionic current through the nanopore, we add $1 \mu \mathrm{l}$ to $5 \mu \mathrm{l}$ of DNA solution (estimated concentration between 100 and $500 \mathrm{ng} / \mu \mathrm{l}$ ) to the cis reservoir of the setup, which contains between $20 \mu \mathrm{l}$ and $50 \mu \mathrm{l}$ of solution and is kept at a negative potential. Figure 4 shows the ionic current versus time at $120 \mathrm{mV}$ bias before and after addition of $11.5 \mathrm{kbp}$ DNA. The nanopore is by far the highest impedance in the electrical circuit, and therefore the magnitude of the current is solely determined by the impedance of the nanopore (typically around $30 \mathrm{M} \Omega$ for a 10 -nm pore). We clearly observe short depressions of the ionic current in the presence of DNA. The ionic current was averaged during $2 \mathrm{~ms}$ before each event to determine the open-pore current. This was done for each event individually to compensate for slow drift of the open-pore current (up to $5 \mathrm{pA}$ per minute). The drift is likely to be caused by shifts in the potential of the $\mathrm{Ag} / \mathrm{AgCl}$ electrodes and concentration changes due to evaporation. All further analysis was performed on currents relative to the open-pore current $I_{\text {base line. }}$. We determined the time duration $\left(t_{\text {dwell }}\right)$ of the event and 

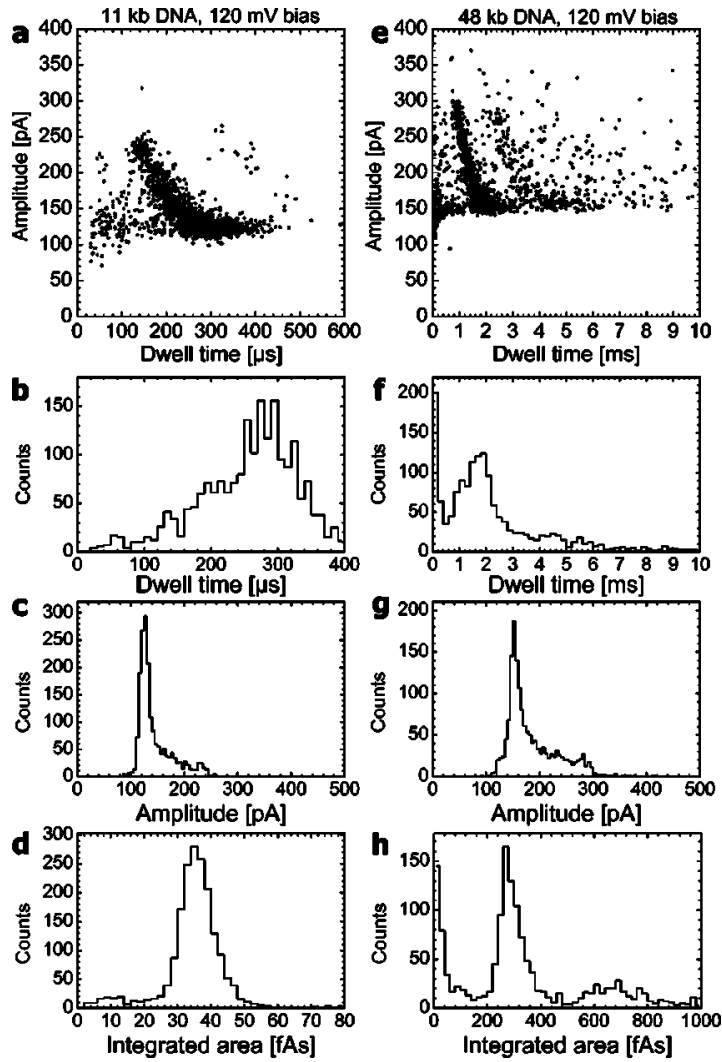

FIG. 5. (a) Event scatter plot of 1855 events recorded at $120 \mathrm{mV}$ bias with $11.5 \mathrm{kbp}$ linear DNA molecules. For each event the dwell time and the average amplitude were determined. Each point in the graph corresponds to a single event. (b) Histogram of observed dwell times for $11.5 \mathrm{kbp}$ DNA. (c) Histogram of observed amplitudes for $11.5 \mathrm{kbp}$ DNA. (d) Histogram of observed areas for $11.5 \mathrm{kbp}$ DNA. (e) Event scatter plot of 1598 events recorded at $120 \mathrm{mV}$ bias with $48.5 \mathrm{kbp} \lambda$-DNA molecules. (f) Histogram of observed dwell times for $48.5 \mathrm{kbp}$ DNA. (g) Histogram of observed amplitudes for $48.5 \mathrm{kbp}$ DNA. (d) Histogram of observed areas for $48.5 \mathrm{kbp}$ DNA.

integrated $\left(I(t)-I_{\text {base line }}\right)$ versus time. This integral is denoted the "area" of the event and is used to calculate the average amplitude $\left[I_{\text {block }}=\right.$ area $\left./ t_{\text {dwell }}\right]$. Thousands of events were analyzed, and the results are plotted in Figs. 5(a) and 5(e), where each point represents a single event. Figures 5(b) and 5(f) show histograms of $t_{\text {dwell }}$ and Figs. 5(c) and 5(g) show histograms of $I_{\text {block }}$ for these two molecules. As is clear from these figures, events are clustered together for both molecules. Both the dwell time and amplitude distributions appear to be quite broad. The two are correlated though: The shorter the dwell time, the higher the amplitude $I_{\text {block}}$. Comparing the results for the two molecules we find a similar distribution of amplitudes for both a peak near $140 \mathrm{pA}$ and a tail up to $300 \mathrm{pA}$. The dwell-time histograms, however, are quite different: For the $48.5 \mathrm{kbp} \lambda$-DNA we find typical dwell times between $1 \mathrm{~ms}$ and $2 \mathrm{~ms}$, about a factor of 7 longer than the 150-350 $\mu$ s observed for the $11.5 \mathrm{kbp}$ molecules.

Closer inspection of the current data suggests that on many occasions, single events consist in fact of one or more plateaus at discrete current levels, as the events for $48.5 \mathrm{kbp}$

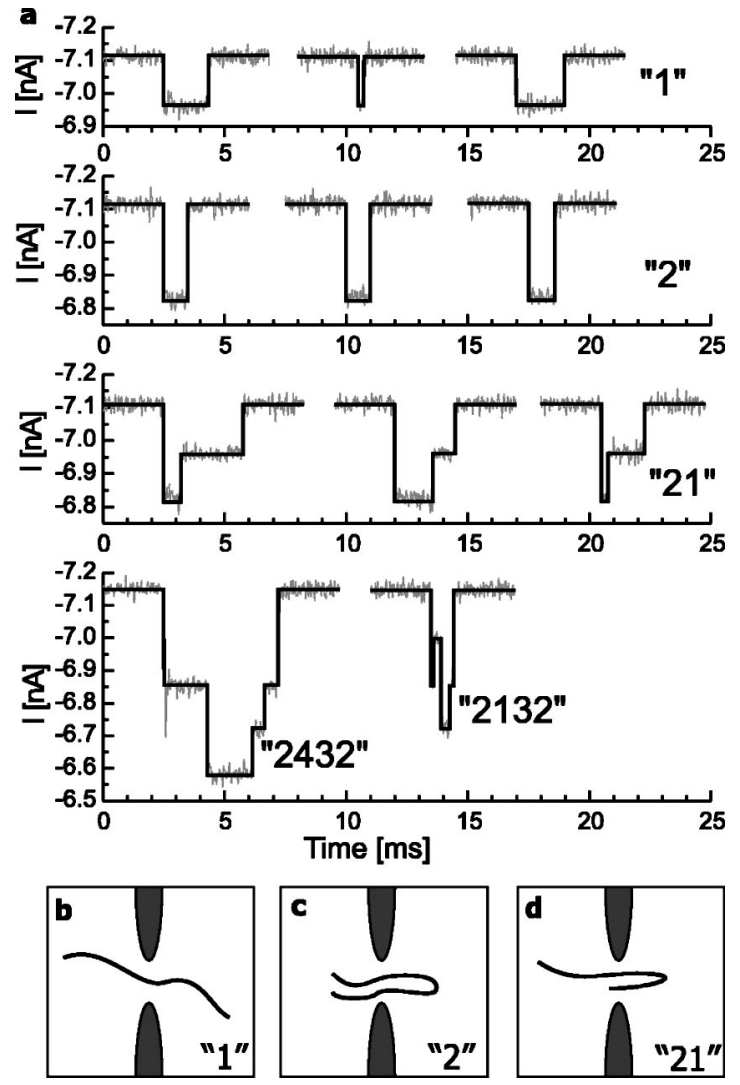

FIG. 6. (a) Examples of recorded events for $\lambda$-DNA. The gray noisy line is the raw data; the black line is the result of a fitting algorithm discussed later. The top panel shows events of type 1 . These events have a single plateau at the first level. Below are events of type 2 . These also have a single plateau, but twice as deep as 1. The third panel shows events with two plateaus: First at the second level and then at the first. These events are labeled as 21 events. Bottom panel: Examples of more complicated event shapes. (b)-(d) Physical interpretations of various event types. Translocation is directed from left to right.

DNA depicted in Fig. 6(a) clearly demonstrate. They can be classified into several types, as discussed below. Discrete current levels are further demonstrated in Fig. 7, where we show a histogram of 5 - $\mu$ s current samples relative to the base line. Note that each count in this histogram corresponds to a single current measurement, not to a single event. A typical recorded time trace lasts about $6 \mathrm{~ms}$ and therefore generates roughly 1200 counts in the histogram. Figure 7 compiles about 1600 time traces, or $2 \times 10^{6}$ counts. Sharp peaks at $0 \mathrm{pA}, 150 \mathrm{pA}, 294 \mathrm{pA}, 434 \mathrm{pA}, 569 \mathrm{pA}$, and $699 \mathrm{pA}$ are evident. The first peak at $0 \mathrm{pA}$ is the open pore current, recorded before and after the event.

The fact that we only observe the current blockades in the presence of DNA proves that they are caused by DNA molecules interacting with the nanopore. We interpret peaks labeled $1-5$ as corresponding to the presence of one or more double strands of DNA inside the pore. Note that we observe events that have up to five ds-DNA within the pore simultaneously. Two effects can cause the presence of more than one double-strand inside the pore: Two or more molecules can translocate at the same time or a single molecule pass the 


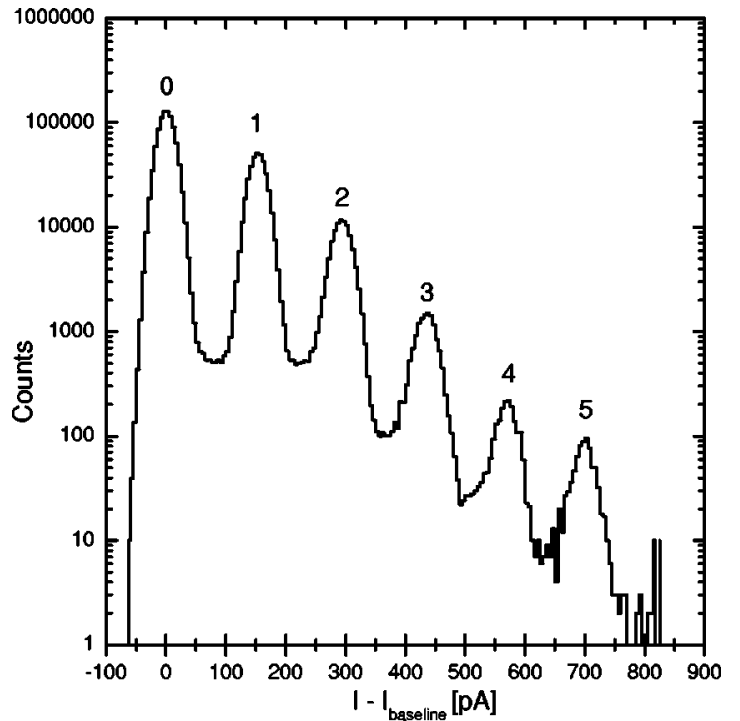

FIG. 7. Histogram of 5- $\mu$ s current samples, obtained from about 1600 recorded time traces, each about $6 \mathrm{~ms}$ long. For each event the base line (open pore) current was determined from the pretrigger part of the measurement. This value was subtracted and a histogram was calculated from all recorded 5 - $\mu$ s samples of the current, relative to the base line.

pore in a folded fashion. We find that the average time between events is around one second, much longer than the average duration, which is on the order of a millisecond or shorter. If we assume that events are not correlated in time, the chance that two events coincide is thus very rare. This is in conflict with the high ratio of higher-order events (up to $50 \%$ of detected events contain a plateau above the 1 level), and thus the folding mechanism appears to be most likely explanation. In Sec. III D, we will interpret the data in terms of folded translocations.

The expected current signal when a DNA molecule is inside the nanopore will depend on the exact geometry of the pore. If we assume that the pore is cylindrical (and ignore edge effects), the current through the open nanopore is $I$ $=\sigma A V / l$, where $\sigma$ is the conductivity of the electrolyte, $A$ is the cross section of the pore, $V$ is the bias voltage, and $l$ is the length of the pore. When a polymer is inside the pore, the relative change in current will be $\Delta I / I=\Delta A / A$, where $\Delta A$ is the cross section of the polymer. Based on this simple model, we expect current blockades of about $4 \%$ for a 2 -nm-diam DNA molecule inside a $10-\mathrm{nm}$ pore. This is in good agreement with the experiments where we find the first discrete plateau in the ionic current depression at about 3\% of the open-pore current. Note that the peaks in Fig. 7 are equidistant, in agreement with the assumption that the current blockade is proportional to the total cross-sectional area of the double strands of DNA.

\section{B. Influence of the bias voltage}

The negatively charged DNA molecules experience a large electrostatic force inside the pore due to the electric field in the pore. This force can be varied by varying the bias
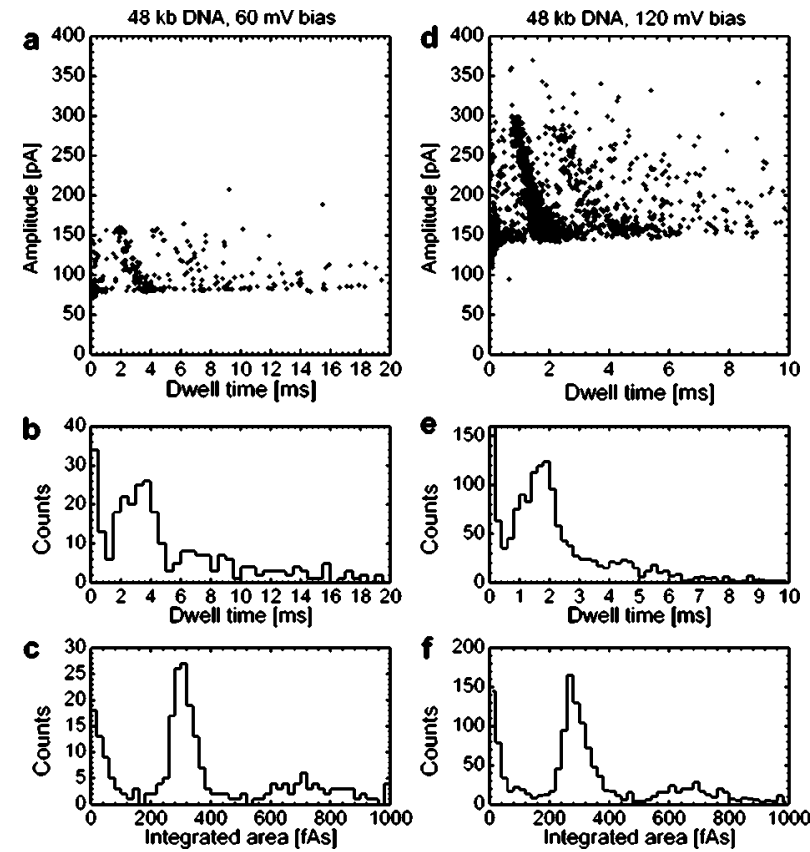

FIG. 8. (a) Event scatter plot of events recorded at $60 \mathrm{mV}$ with 48.5-kbp linear DNA molecules. (b) Histogram of observed dwell times at $60 \mathrm{mV}$. (c) Histogram of the area (integrated signal) of events at $60 \mathrm{mV}$. (d) Event scatter plot of events recorded at $120 \mathrm{mV}$ with 48.5-kbp linear DNA molecules. (e) Histogram of observed dwell times at $120 \mathrm{mV}$. (f) Histogram of the area (integrated signal) of events at $120 \mathrm{mV}$.

voltage that is applied over the pore. To demonstrate the influence of the driving voltage on the current blockades, the following experiment was performed. After collecting the data on $48.5 \mathrm{kbp}$ linear $\lambda$-DNA at a voltage of $120 \mathrm{mV}$ discussed above, we reduced the bias voltage to $60 \mathrm{mV}$, a factor of 2 lower. At this lower voltage, blockades occurred much less frequently. Figure 8(a) shows a scatter plot of dwell time and amplitude of the 307 events collected at this voltage. For comparison the results for events recorded at $120 \mathrm{mV}$ are shown again in Figs. 8(d)-8(f). The general shape of the distribution is similar, but the magnitude of both dwell time and amplitude have changed. We now find amplitudes between $75 \mathrm{pA}$ and $150 \mathrm{pA}$, half of the $150 \mathrm{pA}$ to $300 \mathrm{pA}$ obtained for the $120 \mathrm{mV}$ events. This is explained by the notion that DNA molecules change the ionic conductance of the pore. Therefore, a reduction of the driving voltage by a factor of 2 will lead to a reduction of the measured current by the same factor. The main peak in the dwell time histogram of the $60-\mathrm{mV}$ data is roughly between 2 and $4 \mathrm{~ms}$, a factor of 2 longer than at $120 \mathrm{mV}$. Clearly, the reduced voltage gives rise to slower dynamics. Note that the integrated-area histograms Figs. 8(c) and 8(f) are about equal.

\section{Interpretation of current blockades}

Our results are in good agreement with similar experiments on solid-state nanopores by $\mathrm{Li}$ et al. [3], who found similar results and demonstrated convincingly that current blockades are caused by translocation of DNA through sili- 
con nitride nanopores. We find that the event duration depends strongly on the length of the molecules in solution, which provides strong evidence that the observed blockades of ionic current are caused by translocation of DNA molecules from the cis to the trans side of the pore. It is very unlikely that the events are caused by DNA insertion in the pore without translocation (i.e., molecules are released back into the cis reservoir) because the observed dependence of the residence time on the DNA length is incompatible with this picture. The length dependence will be further demonstrated in an additional experiment on a mixture of DNA, presented later. When we invert the polarity of the applied voltage, current blockades are extremely rare. This observation is also in agreement with the interpretation in terms of translocation, and the rare events that are observed at inverse bias can be attributed to translocation of molecules from the trans to the cis side. It is interesting to note that a much fainter second band of events is apparent in Fig. 5(e) at roughly double the time scale as the first band. We argue that complementary sticky ends present on the $\lambda$-DNA cause occasional binding of two DNA molecules. This would explain the second band of events at longer dwell times compared to the main band.

\section{Analysis of translocation events}

Further analysis of individual events was performed using a dedicated Labview (a graphical programming language from National Instruments) program. Our main aim was to determine the height and duration of the plateaus within an event. The position of the peaks in Fig. 7 is the basis for our event-fitting algorithm to allow sorting and automated analysis on a large number of events. At the position of the peaks in Fig. 7 we define discrete levels labeled from 0 to 5, and for each event we fit all current data to the nearest discrete level. If a certain level lasts shorter than $30 \mu$ s, we discard it and change it to the level directly following it. This procedure is performed because the data was filtered at $10 \mathrm{kHz}$, and consequently levels shorter than $30 \mu$ s are likely to be caused by a slow response of the filter to a level change of more than one "step." Another cause for undesired short events is that occasionally the random noise reaches the threshold for a neighboring level. The fitting procedure reduces each event to a sequence of one or more subevents, where a subevent is characterized by one of the five discrete levels of current blockade and its duration. Figure 6(a) shows the measured signal for a number of events and the result of the described fitting procedure. We group similar events by the sequence of levels of its subevents. Events of type 1, for example, are simple blockades to the first level. Events of type 21 consist of two subevents, first at the second level and subsequently the first level. This labeling allows us to study statistics of subsets of all measured events. Figures 6(b)-6(d) show the physical interpretation of a number of event types.

The sorting algorithm described in the methods section allows us to study well-defined subsets of all measured events. First we take a look at the most simple events, those of type 1 . These events are straightforwardly interpreted as simple linear translocations of molecules from one end to the
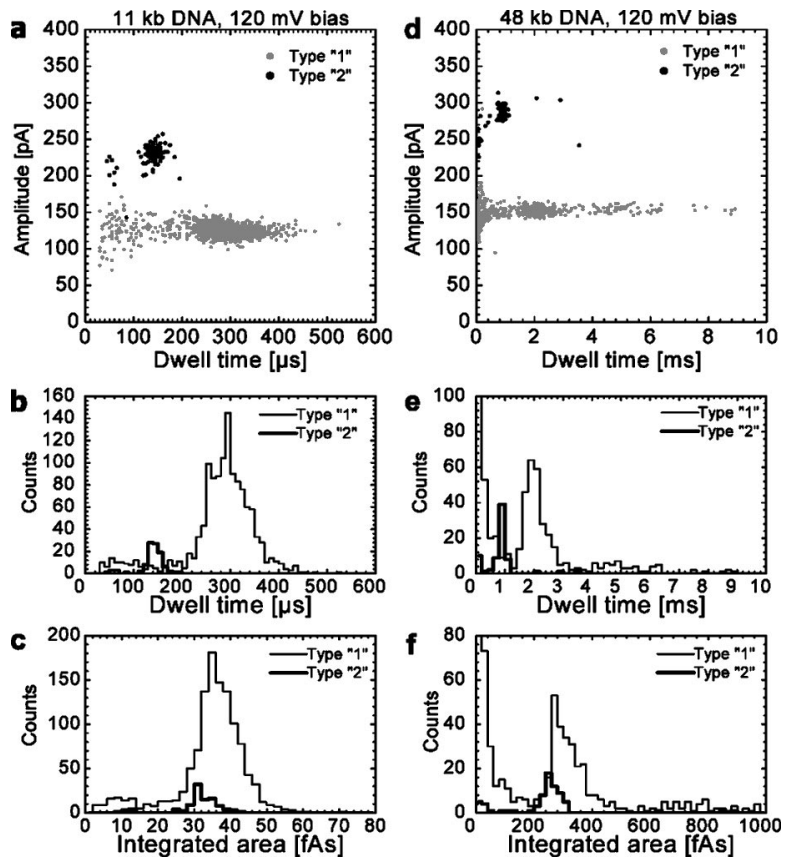

FIG. 9. (a) Event scatter plot of type-1 (gray) and type-2 (black) events recorded at $120 \mathrm{mV}$ with 11.5 -kbp linear DNA molecules. (b) Histogram of observed dwell times for 1 and 2 events. (c) Histogram of the area (integrated signal) of events of type 1 and 2. (d) Event scatter plot of type-1 (gray) and type-2 (black) events recorded at $120 \mathrm{mV}$ with 48.5 -kbp linear DNA molecules. (e) Histogram of observed dwell times for 1 and 2 events. (f) Histogram of the area (integrated signal) of events of type 1 and 2 .

other, without any folding. Figures 9(a) and 9(d) show a scatter plot of events of type 1 for the 11.5-kbp and 48.5-kbp data sets, respectively (plotted in grey). In Figs. 9(b) and 9(e) the histograms of $t_{\mathrm{dwell}}$ are plotted, and in Figs. 9(c) and 9(f) we show a histogram of the areas. In a similar way, we analyzed events of type 2 , which are interpreted as molecules folded exactly in the middle. It should be noted that $\lambda$-DNA has single-strand "sticky ends" at both ends and that these ends can bind to form circular DNA. Translocation events from circular molecules are expected to look identical to molecules folded exactly halfway (see also the data on circular DNA discussed below). The 2 results were plotted in black in the same graphs as the type- 1 events for comparison. For both data sets, we fitted a Gaussian to the peaks in the dwell time and the area histograms. The results are shown in Table I. We see that, for both molecules, the dwell time of 2 events equals about half the time of the 1 events. Additionally, the areas are almost constant: If we look at the integrated area for both types of events displayed in Figs. 9(c) and 9(f), we find a difference of less than $10 \%$ between type 1 and 2. Apparently, the average velocity of the molecules is roughly identical for folded and straight DNA translocations.

Another type of event that frequently occurs is the 21 event-i.e., blockades where we first observe a deep blockade at the second level, followed by a plateau at the first level. These events fall in between the 1 and 2 events in the scatter plot [compare Figs. 9(a) and 9(d) to Figs. 8(a) and $8(\mathrm{~d})$ ]. Now we define $t_{1}$ as the duration of the first subevent 
TABLE I. Summary of experimental results: Events detected for $11.5 \mathrm{kbp}$ and $48.5 \mathrm{kbp}$ DNA were sorted to type. The peaks in the dwell time and area distributions were fitted to a Gaussian to determine the mean and standard deviation $\sigma$.

\begin{tabular}{|c|c|c|c|c|c|c|}
\hline \multicolumn{7}{|c|}{$11.5 \mathrm{kbp}$ DNA $(N=1855)$} \\
\hline Type & Number & $\begin{array}{l}\% \text { of } \\
\text { total }\end{array}$ & $\begin{array}{c}t_{\mathrm{dwell}} \\
\text { mean } \\
(\mu \mathrm{s})\end{array}$ & $\begin{array}{c}t_{\text {dwell }} \\
\sigma \\
(\mu \mathrm{s})\end{array}$ & $\begin{array}{l}\text { Area } \\
\text { mean } \\
(\mathrm{fAs})\end{array}$ & $\begin{array}{c}\text { Area } \\
\sigma \\
\text { (fA s) }\end{array}$ \\
\hline 1 & 1186 & 63.9 & 292 & 38 & 36.5 & 4.8 \\
\hline 2 & 102 & 5.5 & 143 & 12 & 32.1 & 2.8 \\
\hline 21 & 513 & 27.7 & a & a & 35.0 & 4.0 \\
\hline 12 & 4 & 0.2 & $\mathrm{~b}$ & $\mathrm{~b}$ & $\mathrm{~b}$ & $\mathrm{~b}$ \\
\hline \multicolumn{7}{|c|}{$48.5 \mathrm{kbp}$ DNA $(N=1598)$} \\
\hline Type & Number & $\begin{array}{l}\% \text { of } \\
\text { total }\end{array}$ & $\begin{array}{c}t_{\mathrm{dwell}} \\
\text { mean } \\
(\mathrm{ms})\end{array}$ & $\begin{array}{c}t_{\text {dwell }} \\
\sigma \\
(\mathrm{ms})\end{array}$ & $\begin{array}{l}\text { Area } \\
\text { mean } \\
(\mathrm{fAs})\end{array}$ & $\begin{array}{c}\text { Area } \\
\sigma \\
\text { (fA s) }\end{array}$ \\
\hline 1 & 633 & 39.6 & 1.96 & 0.28 & 298 & 45 \\
\hline 2 & 72 & 4.5 & 0.90 & 0.12 & 258 & 28 \\
\hline 21 & 683 & 42.7 & a & a & 285 & 34 \\
\hline 12 & 4 & 0.3 & b & $\mathrm{b}$ & $\mathrm{b}$ & b \\
\hline
\end{tabular}

${ }^{\mathrm{a}}$ Wide non-Gaussian distribution.

${ }^{\mathrm{b}}$ Too few events were detected for reliable fitting.

(at level 2) and $t_{2}$ as the duration of the second subevent (at level 1). Figures $9(\mathrm{~b})$ and $9(\mathrm{e})$ show $t_{2}$ versus $t_{1}$ for $11.5 \mathrm{kbp}$ and $48.5 \mathrm{kbp}$ DNA, respectively. For both molecules, we observe a linear relation between the two. We interpret these 21 events in the following way: DNA passes the pore in a folded state. First, the double part of the molecule passes the pore, followed by the residual linear part of the molecule; see Fig. $6(d)$. It is interesting to note that events of type 12 are rare; see Table I. It thus appears that once translocation starts in a linear fashion, capture of the other end in the pore is quite unlikely.

In Table I, we summarize our findings. It appears that more than $85 \%$ of events can be identified under the simple assumption that a molecule passes the pore either linearly or with a single fold. We now estimate the position of the fold along the molecule from the measured event. We define the capture position as $x / L$, where $x$ is the distance from the folding point to the nearest end of the molecule and $L$ is the total contour length. Linear, unfolded translocations of type 1 have a fold position of 0 , and type 2 translocations a fold position of 0.5 . To estimate the capture position from type 21 we assume that the first "double" plateau is related to the passage of two double strands with length $x$ in parallel and that the following single blockade is the residual part of the molecule with length $(L-2 x)$. Assuming constant velocity, the capture position can now be estimated as $t_{1} /\left(2 t_{1}+t_{2}\right)$. In the histogram in Figs. 10(c) and 10(f) we show the result of this analysis, compiling results for 1, 2, and 21 type events, for both $11.5 \mathrm{kbp}$ and $48.5 \mathrm{kbp}$ DNA, respectively. Disregarding the positions 0 and 0.5 for the moment, we see a distribution that is roughly uniform within the experimental error. Note that the absence of events with a folding location around 0.1 in the case of $11.5 \mathrm{kbp}$ DNA is caused by the finite time resolution of the electronics. Events of type 21 where the double part occupies the pore for less than about $50 \mu$ s are likely to be detected as 1 events.

Since DNA is coiled up randomly in solution, one would expect that the distribution of fold locations is completely flat. We see however that unfolded molecules (capture position 0) appear about 10 times more often than expected. Apparently, it is more likely to initiate the translocation of a DNA molecule at one of its ends than at another position. We speculate that the kink in the DNA that is necessary for entry in a folded state causes a considerable energy barrier in the translocation process. This may explain the difference in frequency for folded and unfolded translocations.

Additionally, we find that for $48.5 \mathrm{kbp}$ DNA, a fold position around 0.5 is about a factor of 2 more likely than other nonzero folding positions. This feature can be accounted for by the presence of a small fraction of $\lambda$-DNA molecules in a circular state. $\lambda$-DNA contains complementary "sticky ends" of about ten bases at both ends of the molecule, which promote the formation of circular DNA. We do not observe this feature for the $11.5 \mathrm{kbp}$ sample, since the restriction enzyme that linearizes the vector leaves sticky ends of only four bases, which are too short for any detectable fraction of circular DNA. Complementary sticky ends of $\lambda$-DNA also explain the pronounced second band of events in Fig. 5. We attribute this to events that are caused by two $\lambda$-DNA molecules bound together. 

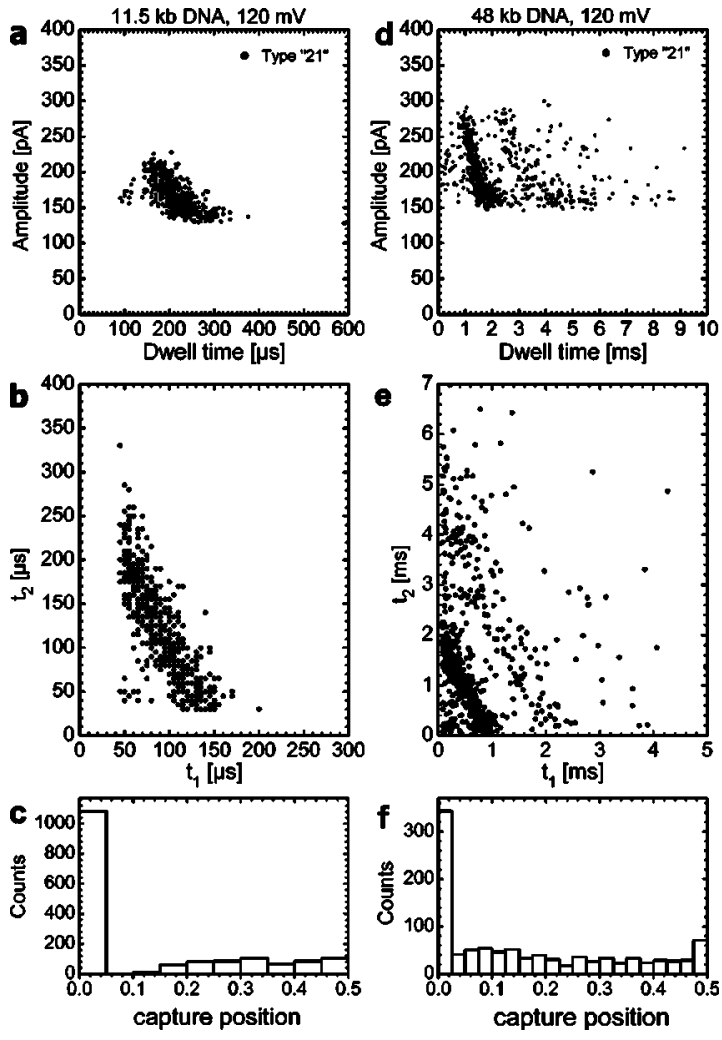

FIG. 10. (a) Event scatter plot of type-21 events recorded at $120 \mathrm{mV}$ for $11.5 \mathrm{kbp}$ DNA. (b) The duration of the first $\left(t_{1}\right)$ and second $\left(t_{2}\right)$ plateau within the 21 type events were determined and plotted as a scatter plot. (c) Calculated capture position for $11.5 \mathrm{kbp}$ DNA. (d) Event scatter plot of type-21 events recorded at $120 \mathrm{mV}$ for $48.5 \mathrm{kbp}$ DNA. (e) The duration of the first $\left(t_{1}\right)$ and second $\left(t_{2}\right)$ plateau within the 21 type events were determined, and plotted as a scatter plot. (f) Calculated capture position for $48.5 \mathrm{kbp}$ DNA.

\section{E. Circular DNA translocation events}

As a further test of the folding model, we conducted another experiment with circular double-strand DNA of $11.5 \mathrm{kbp}$ length and an 8-nm pore. Circular DNA can exist in various isomers, since the loop can contain a number of different "twists" [8]. This supercoiling effect has a large effect of the structure of the molecule. To circumvent these effects, we worked with double-strand DNA with one nick: The backbone of one of the two strands contains a single break, allowing supercoils to relax. This way we can be sure to have DNA molecules in an identical state. Repeating our nanopore experiment with a bias voltage of $120 \mathrm{mV}$ on this nicked circular DNA, we again observe clear blockage events. With the same data analysis described earlier, $t_{\text {dwell }}$ and $I_{\text {block }}$ were determined for all events, and the results are plotted in Fig. 11. The distribution of events appears to be qualitatively different from the distribution observed for linear molecules. Instead of a band of events, we detect primarily type- 2 events. The average dwell time is around $190 \mu \mathrm{s}$ and the amplitude is around $120 \mathrm{pA}$. The value for the dwell time agrees reasonably well with the value of $143 \mu$ s for type- 2 events for the linear molecules. The value for the amplitude, however, significantly deviates from the $240 \mathrm{pA}$ observed for

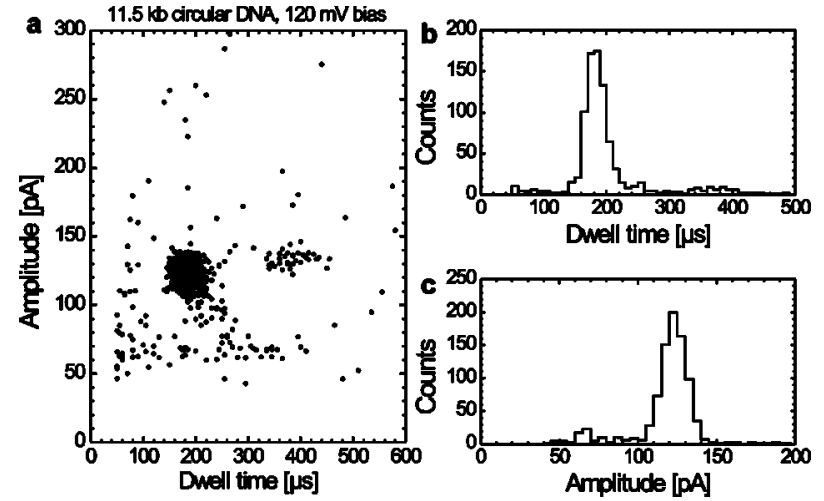

FIG. 11. (a) Event scatter plot of events recorded at $120 \mathrm{mV}$ with 11.5-kbp circular DNA molecules. (b) Histogram of observed dwell times. (c) Histogram of the amplitudes.

the linear molecules. We attribute this difference to differences between the pores that were used. The diameter of the pore was $8 \mathrm{~nm}$ in this experiment. The length of the pores, however, is not well known and a sizable difference could explain the difference in amplitudes.

\section{F. Nanopore experiment on a polydisperse DNA mixture}

The observation that the dwell time increases with increasing length of DNA suggests that nanopores can be used to determine the lengths of unknown fragments, analogous to traditional gel electrophoresis. As a first evaluation, we analyzed translocation events recorded on a mixture of DNA fragments with known sizes. A commercially available mixture of DNA markers for gel electrophoresis was tested. The mixture contains fragments that remain after incubation of $\lambda$-DNA with the HindIII restriction enzyme. The mixture contains fragments of sizes (1) $23130 \mathrm{bp}$, (2) $9416 \mathrm{bp}$, (3) 6557 bp, (4) 4361 bp, (5) 2322 bp, (6) 2027 bp, (7) 564 bp, and (8) $125 \mathrm{bp}$. Because linear $\lambda$-DNA contains single-strand sticky ends, fragments (1) and (4) can bind together to form effectively a 27491 - bp fragment. We performed a gel electrophoresis experiment in order to determine the amount fragments bound in our sample. Figure 12 shows the gel results for the mixture as supplied (left lane) and after a short heating to about $60{ }^{\circ} \mathrm{C}$ (right lane). We find that indeed fragments (1) and (4) are bound together almost completely in the material before heating. We conclude that the following fragment sizes are present in the material as supplied: 27491 bp (1+4), 9416 bp (2), 6557 bp (3), 2322 bp (5), 2027 bp (6), 564 bp (7), and 125 bp (8).

A nanopore translocation experiment was carried out in the manner reported in the preceding sections using the DNA mixture as supplied, without heating. Events were analyzed with the same protocol as discussed in the materials and methods section. First, we look at the distribution of dwell times for type-1 events (linear translocations without folding). The histogram shown in Fig. 13 shows the result of this analysis. Quite clearly we observe three peaks at $1 \mathrm{~ms}$, $260 \mu \mathrm{s}$, and $160 \mu \mathrm{s}$, and we identify these peaks as caused by fragments $(1+4)$, fragment (2), and fragment (3), respectively. Figure 13(b) shows an integrated-area histogram from 


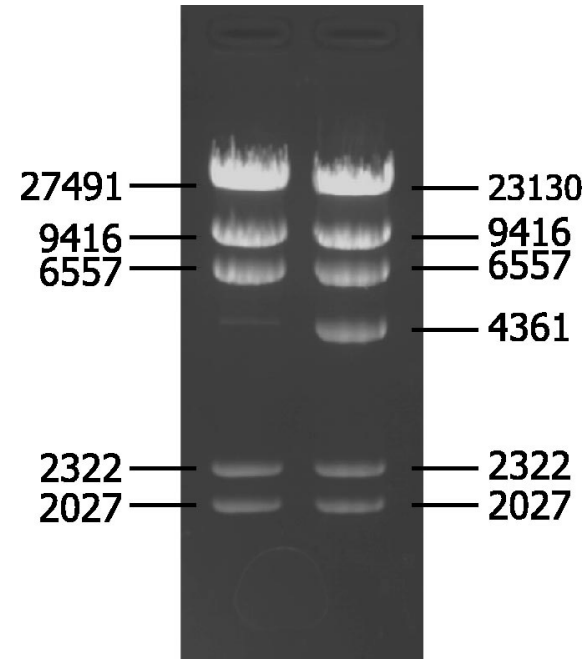

FIG. 12. Gel electrophoresis on the DNA mixture. The left lane shows the DNA mixture as supplied; the right lane contains the same mixture that was heated to about $60{ }^{\circ} \mathrm{C}$ and cooled shortly before loading. The nanopore experiments were performed on material as supplied. Note that without the heat treatment the fragments of 4361 bases and 23130 bases are bound together to a single molecule of 27491 bases.

all measured events, again showing three clear peaks.

\section{DISCUSSION}

From the DNA-mixture experiment, it is clear that the longer the DNA, the longer the translocation time. To make this claim more quantitative, we compile the average dwelltimes observed for all linear type-1 translocations in Fig. 14. We find a nonlinear behavior for the translocation time $t_{\text {dwell }}$ as a function of the length $L$. The straight line on the log-log

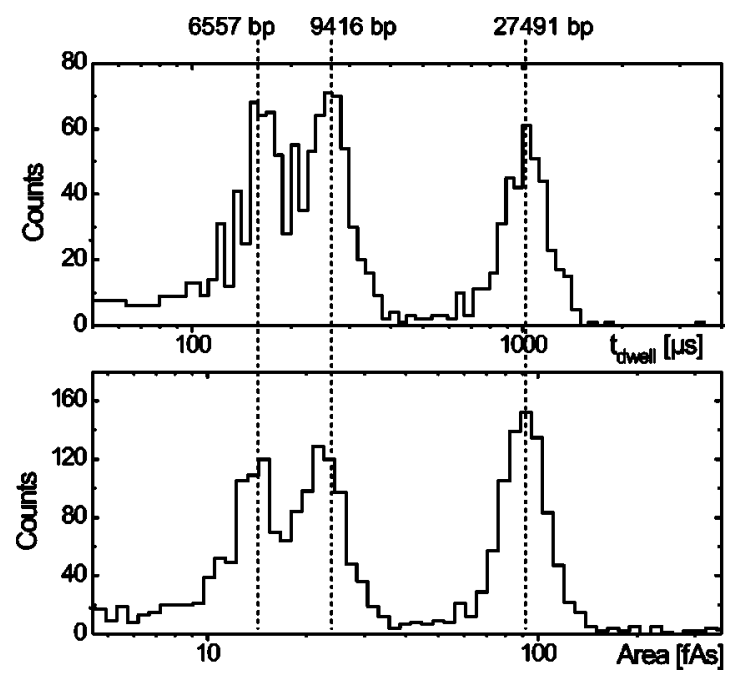

FIG. 13. Top panel: histogram of dwell times of type-1 events obtained with a mixture of DNA molecules. The four peaks can be identified with the longest fractions of DNA present in the mixture. Bottom panel: histogram of the areas measured for all detected events.

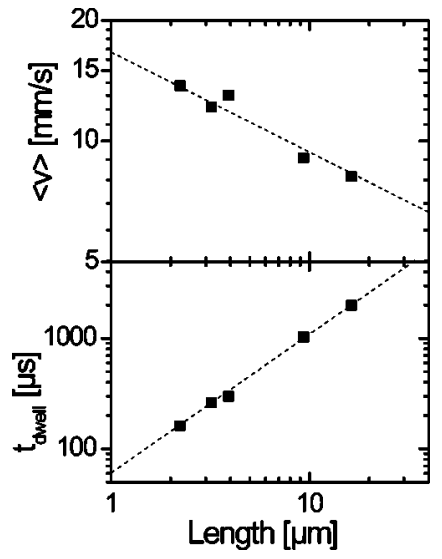

FIG. 14. Average velocity and dwell time vs DNA length. $t_{\text {dwell }}$ was taken as the most probable dwell time for a molecule-i.e., the location of the peak in the dwell time histogram. The average velocity $\langle v\rangle$ is calculated as $L / t_{\mathrm{dwell}}$.

plot shows a power-law $t_{\text {dwell }} \sim L^{\alpha}$ fit to the data, with an exponent $\alpha$ of 1.26. Figure 14 also shows the average translocation velocity calculated as $\langle v\rangle=L / t_{\mathrm{dwell}}$ for all molecules. We now compare these results to published data on singlestrand DNA and RNA translocation through $\alpha$-hemolysin. First, there is a large difference in translocation velocity. At a driving voltage of $120 \mathrm{mV}$, nucleic acids thread the $\alpha$-hemolysin pore at a rate of about 1 base per microsecond [9], equivalent to about $0.5 \mathrm{~mm} / \mathrm{s}$. This is much slower than the $10 \mathrm{~mm} / \mathrm{s}$ that we observe for double-strand DNA. This effect can be explained by a difference in effective friction between the polymer and pore. $\alpha$-hemolysin appears to have strong specific interactions with the passing polymers. In this system, the translocation time scales linearly with length $[1,5]$. It thus appears that the DNA inside the nanopore dictates the speed of translocation. In contrast, the solid-state nanopores used in this research were relatively wide compared to the double-strand DNA. We argue that this leads to a negligible drag force inside the nanopore and thus to high translocation velocities. In this regime, we expect that the primary drag force is the hydrodynamic drag on the full length of DNA. The observed decrease of translocation velocity with length is in agreement with this assumption. A scaling model for polymer translocation through solid-state nanopores will be published elsewhere [10].

\section{CONCLUSIONS}

To summarize, we have observed single-molecule DNA translocation events through silicon oxide nanopores fabricated by our new TEM shrinking technique. Using nanopores of 8-10 nm diameter, we have detected translocation events for DNA fragments of $11.5 \mathrm{kbp}$ and $48.5 \mathrm{kbp}$. The distribution of both the event duration and their amplitude can be qualitatively well understood if we take folding of the molecules into account. A detailed analysis of the exact shape of the event can then be used to estimate the fold position. In the future, similar techniques may be applied to address the folding of RNA or even polypeptides. 
Moreover, we demonstrate that a nanopore-based detector can be used analyze polydisperse mixtures of DNA, with fragment sizes from $6.5 \mathrm{kbp}$ to about $27 \mathrm{kbp}$. In this experiment we analyze the translocation of about 2500 individual DNA molecules $\left(3 \times 10^{-14} \mathrm{~g}\right)$ and find a clear separation in length. This result proves the remarkable sensitivity of nanopores, although the resolving power in this first device is not yet up to standards set by traditional gel electrophoresis.

Finally, analysis of all translocation data shows that for unfolded translocations, the duration $t_{\text {dwell }}$ scales with the length $L$ as $t_{\mathrm{dwell}} \sim L^{\alpha}$, with an exponent $\alpha$ of 1.26. This behavior is in disagreement with existing models for translocation and suggests that a different mechanism dominates the translocation dynamics in these experiments. Understanding the underlying physics of the translocation process is relevant for the development of future nanopore-based detectors.

\section{ACKNOWLEDGMENTS}

We would like to thank the groups of D. Branton and J.A. Golovchenko at Harvard University for their hospitality and advice on nanopore experiments. We also thank John van Noort, Peter Veenhuizen, Nynke Dekker, Dick Korbee, and Kees Storm for their support and discussions. This research was financially supported by the Dutch Foundation for Fundamental Research on Matter (FOM) and the Dutch Organisation for Scientific Research (NWO). J.H.C. is grateful to the Netherlands Institute for Metals Research (NIMR) for financial support under Project No. MC4.98047.
[1] J. J. Kasianowicz, E. Brandin, D. Branton, and D. W. Deamer, Proc. Natl. Acad. Sci. U.S.A. 93, 13770 (1996).

[2] J. L. Li, D. Stein, C. McMullan, D. Branton, M. J. Aziz, and J. A. Golovchenko, Nature (London) 412, 166 (2001).

[3] J. L. Li, M. Gershow, D. Stein, E. Brandin, and J. A. Golovchenko, Nat. Mater. 2, 611 (2003).

[4] A. J. Storm, J. H. Chen, X. S. Ling, H. W. Zandbergen, and C. Dekker, Nat. Mater. 2, 537 (2003).

[5] A. Meller, L. Nivon, and D. Branton, Phys. Rev. Lett. 86, 3435 (2001).
[6] R. W. DeBlois and C. P. Bean, Rev. Sci. Instrum. 41, 909 (1970).

[7] H. Chang, F. Kosari, G. Andreadakis, M. A. Alam, G. Vasmatzis, and R. Bashir, Nano Lett. 4, 1551 (2004).

[8] B. Alberts and D. Bray, Molecular Biology of the Cell (Garland Publishing, New York, 1994).

[9] A. Meller, A. Nivon, A. Brandin, J. Golovchenko, and D. Branton, Proc. Natl. Acad. Sci. U.S.A. 97, 1079 (2000).

[10] A. J. Storm, C. Storm, J. H. Chen, H. W. Zandbergen, J. F. Joanny, and C. Dekker, Nano Lett. (to be published). 